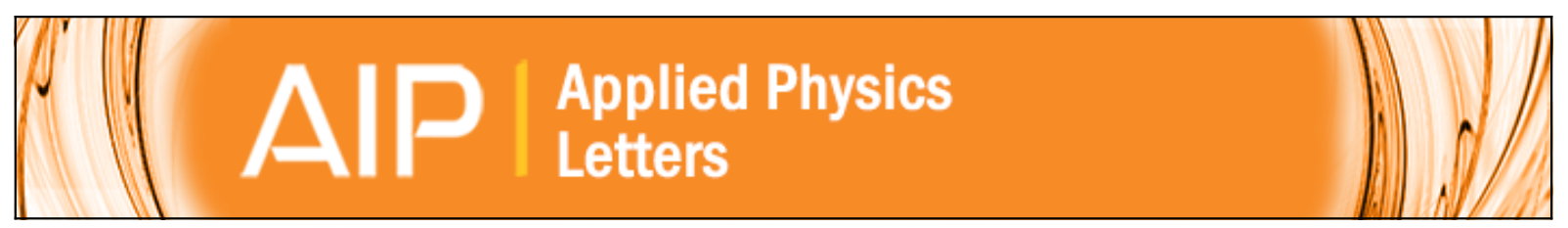

\title{
Phase transformation as the single-mode mechanical deformation of silicon
}

S. Wong, B. Haberl, J. S. Williams, and J. E. Bradby

Citation: Applied Physics Letters 106, 252103 (2015); doi: 10.1063/1.4923205

View online: http://dx.doi.org/10.1063/1.4923205

View Table of Contents: http://scitation.aip.org/content/aip/journal/apl/106/25?ver=pdfcov

Published by the AIP Publishing

\section{Articles you may be interested in}

Temperature-dependent mechanical deformation of silicon at the nanoscale: Phase transformation versus defect propagation

J. Appl. Phys. 117, 205901 (2015); 10.1063/1.4921534

Temperature dependent deformation mechanisms in pure amorphous silicon

J. Appl. Phys. 115, 113511 (2014); 10.1063/1.4869136

Deformation mechanisms in silicon nanoparticles

J. Appl. Phys. 109, 063534 (2011); 10.1063/1.3552985

Effect of hydrogen on nanoindentation-induced phase transformations in amorphous silicon

J. Appl. Phys. 106, 123511 (2009); 10.1063/1.3267853

Rate-dependent phase transformations in nanoindented germanium

J. Appl. Phys. 105, 126101 (2009); 10.1063/1.3151967

\section{AlP $\left.\right|_{\text {APL Photonics }}$}

APL Photonics is pleased to announce Benjamin Eggleton as its Editor-in-Chief

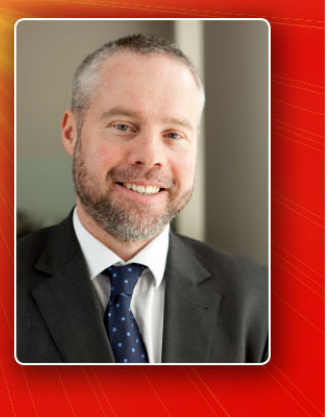




\title{
Phase transformation as the single-mode mechanical deformation of silicon
}

\author{
S. Wong, ${ }^{1, \text { a) }}$ B. Haberl, ${ }^{2}$ J. S. Williams, ${ }^{1}$ and J. E. Bradby ${ }^{1}$ \\ ${ }^{1}$ Department of Electronic Materials Engineering, Research School of Physics and Engineering, \\ Australian National University, Canberra 0200, Australia \\ ${ }^{2}$ Chemical and Engineering Materials Division, Neutron Sciences Directorate, Oak Ridge National \\ Laboratory, Oak Ridge, Tennessee 37781, USA
}

(Received 5 May 2015; accepted 17 June 2015; published online 25 June 2015)

\begin{abstract}
The metastable body-centered cubic (bc8) and rhombohedral (r8) phases of silicon that are formed after the nanoindentation of diamond cubic silicon exhibit properties that are of both scientific and technological interest. This letter demonstrates that large regions of these phases can be readily formed from crystalline silicon via nanoindentation with minimal damage to the surrounding crystal. Cross-sectional transmission electron microscopy is used to show that volumes of these phases $6 \mu \mathrm{m}$ wide and up to $650 \mathrm{~nm}$ deep can be generated using a symmetrical spherical tip of $\sim 21.5 \mu \mathrm{m}$ diameter. This result indicates that the use of large symmetrical spherical tips result in highly hydrostatic conditions that can favor the single phase transformation mode without extensive damage to the surrounding crystalline regions that are observed in previous studies. C 2015 AIP Publishing LLC.
\end{abstract}

[http://dx.doi.org/10.1063/1.4923205]

Silicon, in its most common diamond cubic (dc) phase, forms the basis for a vast array of devices. ${ }^{1,2}$ However, additional metastable phases of Si can also be formed after application of high pressure, such as the body-centered cubic phase (bc8) and the rhombohedral phase ( $(\mathrm{r} 8){ }^{3-5}$ A structure that contains both the bc 8 and $\mathrm{r} 8$ phases and which is stable at ambient conditions can be formed via nanoindentation. ${ }^{6-8}$ This bc $8 / \mathrm{r} 8$ structure can be easily integrated into existing Si-based technology ${ }^{9}$ and is of considerable interest as it contains the semiconducting $\mathrm{r} 8$ phase which may have superior properties to $\mathrm{dc}-\mathrm{Si}$ in some photovoltaic applications. ${ }^{5,10,11}$ Indeed, r8-Si has been predicted to have a greater optical absorption coefficient than dc-Si across the solar spectrum. ${ }^{10}$ This naturally leads to a desire to exploit this phase in possible device applications. Furthermore, the r8 structure obtained following nanoindentation (or more specifically, the mixed bc $8 / \mathrm{r} 8$ structure) has been shown to behave as a narrow band-gap semiconductor with both p-type and n-type dopant activation. ${ }^{12}$

Under near-hydrostatic pressure in a diamond anvil cell (DAC), dc-Si transforms to the metallic beta-tin ( $\beta$-Sn) phase at $\sim 11 \mathrm{GPa}^{3}{ }^{3}$ Upon decompression, this dc to metallic transition is not reversible, and instead, the structure transforms first to the $\mathrm{r} 8$ phase followed by the nucleation of the bc 8 phase. From such DAC studies, the r8 structure is known to be stable to $\sim 2 \mathrm{GPa}^{5}{ }^{5}$ while the bc 8 structure is reported to be stable in ambient conditions. ${ }^{13}$ A similar series of transformations are induced if localized pressure is applied with an indenter. ${ }^{7}$ On unloading, it has been widely reported that $(\beta$-Sn)-Si under the indenter transforms into a mixture of bc8 and $\mathrm{r} 8{ }^{14-18}$ Although there have been some reports of the respective percentages of bc8 and $\mathrm{r} 8$ phases present using Raman spectroscopy, ${ }^{18,19}$ this is problematic as the Raman phonon frequencies of these phases are very close and contain several overlapping peaks. ${ }^{20}$ Furthermore, the Raman

\footnotetext{
${ }^{\text {a) }}$ Author to whom correspondence should be addressed. Electronic mail: Sherman.Wong@anu.edu.au
}

cross-sections are not well known for each of the phases. Thus, this current study refers to the 'bc $8 / \mathrm{r} 8$ structure' formed after nanoindentation.

Previously, indentation-induced micro-scale regions of the $\mathrm{bc} 8 / \mathrm{r} 8$ structure were always found to co-exist with large concentrations of crystalline defects (such as $\operatorname{slip}^{21,22}$ and dislocations $^{23}$ ) underneath the surface transformed zones. ${ }^{14,18,22,24}$ This study shows that, under certain indentation conditions with spherical tips, some residual impressions can display large volumes of the $b c 8 / \mathrm{r} 8$ structure while avoiding significant damage to the underlying crystal. The indentation-induced phase transformed regions are examined using Raman microspectroscopy, scanning electron microscopy (SEM), and crosssectional transmission electron microscopy (XTEM).

All indentations were prepared with a TI 950 Hysitron Triboindenter fitted with a 3D Omniprobe ${ }^{\mathrm{TM}}$ using a diamond spherical indenter of $\sim 21.5 \mu \mathrm{m}$ diameter, which is loaded onto a $\mathrm{Cz}$-grown $\mathrm{Si}(100)$ substrate. The tip was loaded at $10 \mathrm{mN} / \mathrm{s}$ and unloaded at $3.3 \mathrm{mN} / \mathrm{s}$ for all indentations. Arrays of $20 \times 2$ indents where made up to a maximum load of $500 \mathrm{mN}$ and $750 \mathrm{mN}$ (in an attempt to account for the stochastic nature of indentation). The simulation package Elastica ${ }^{\circledR 25}$ was utilized to calculate the expected indentationinduced pressure distribution in dc-Si. A Renishaw 2000 system with a $632.8 \mathrm{~nm}$ wavelength laser was used to measure the Raman signature from each residual indent. A FEI Helio 600 NanoLab dual-beam FIB system was employed to both create cross-sections of the residual indent as well as to obtain SEM images with the electron beam column operating at $3 \mathrm{kV}$. Due to the inherent geometry associated with the 'H-bar' cross-section method used, ${ }^{26}$ all cross-sections are aligned to the (010) plane at which the sample was originally cleaved from the wafer. XTEM imaging was performed with a Philips CM 300 at $300 \mathrm{kV}$.

A SEM image of the indentation tip used in this study is shown in Fig. 1(a). The tip can be seen to have a smooth surface and a diameter of $\sim 21.5 \mu \mathrm{m}$. The symmetrical shape of this particular tip is crucial for uniform radial pressure 
distribution, and hence a small hydrostatic pressure gradient from the center of tip contact, into the surrounding material. $^{22,27-30}$ Figure 1(b) shows a typical SEM image of a residual impression after indentation with this tip to a maximum load of $500 \mathrm{mN}$. No cracking of the surrounding surface is observed, and the residual indent has a diameter of $\sim 6 \mu \mathrm{m}$. Figure 1(c) shows an Elastica ${ }^{\circledR}$ simulation of the hydrostatic pressure induced in such an indent. The outer (pink) contour line shows the region experiencing $11 \mathrm{GPa}$ pressure (the pressure at which the dc-Si to $(\beta-\mathrm{Sn})-\mathrm{Si}$ transformation is expected to occur), with the pressure increasing as the contour lines darken toward the middle of the indent. There is excellent agreement between the plastically deformed area observed via SEM and the region exceeding the critical pressure for phase transformation predicated via Elastica ${ }^{\circledR}$ (i.e., circular in shape and $6 \mu \mathrm{m}$ in diameter). It should be noted that although the simulation shows regions in the center of the indent experiencing pressures up to $19 \mathrm{GPa}$. This would not be practically achieved due to the material undergoing plastic deformation before that pressure could be induced and the fact that the program Elastica ${ }^{\circledR}$ only models the elastic deformation and does not take into account the transition to plastic deformation.

Interestingly, Fig. 1(b) also shows that there is no significant extrusion of the material formed around the sides of the indent. This is in contrast to previous studies that have shown extrusions around indents in $\mathrm{Si}$ that is thought to be caused by the transformation to the ductile $\beta$-Sn phase on loading. ${ }^{22,31}$ We suggest that no such extrusions are observed in this study due to the spherical uniformity of the indenter used. The following calculation supports this claim. The contact radius $\left(r_{c}\right)$ for a spherical indenter is given by

$$
r_{c}=\sqrt{r_{t i p}^{2}-\left(r_{t i p}-h\right)^{2}}
$$

where $r_{\text {tip }}$ is $10.8 \mu \mathrm{m}$ [as can be seen from Fig. 1(a)] and $h$ is the penetration depth. For example, for a maximum force of

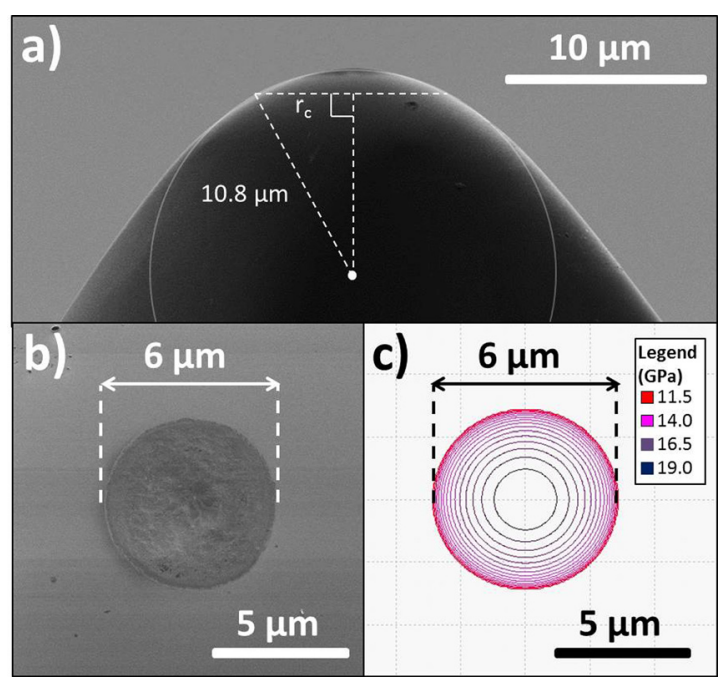

FIG. 1. (a) SEM image of the $21.5 \mu \mathrm{m}$ diameter spherical indentation tip used in this study. (b) A typical residual indent after indentation using this tip to a maximum load of $500 \mathrm{mN}$ and (c) the Elastica simulation of the predicted phase transformed region [i.e., areas experiencing $\geq 11 \mathrm{GPa}$ of hydrostatic pressure required for transformation to $(\beta-\mathrm{Sn})-\mathrm{Si}]$.
$500 \mathrm{mN}$, the indentation load/unload data show that the indenter penetrates $\sim 900 \mathrm{~nm}$ into the surface [see Fig. 3(c)]. Thus, the tip has a contact radius $r_{c} \simeq 4.3 \mu \mathrm{m}$ under these conditions. This is significantly greater than the $3 \mu \mathrm{m}$ radius of the plastic (transformed) region. Therefore, it is clear that the contact area of the indenter is sufficient to fully constrain the transformed region between the indenter and underlying substrate during indentation, suppressing the extrusion of material previously observed by other studies.

A bright-field (BF) XTEM image of a residual indent impression to a maximum load of $500 \mathrm{mN}$ is shown in Fig. 2(a). This image shows a phase transformed circular segment $\sim 6 \mu \mathrm{m}$ wide with a maximum depth of $\sim 650 \mathrm{~nm}$. The dark bands seen in this image under the transformed region are a result of bend contrast caused by residual stress in the thinned sample. ${ }^{32}$ It can be seen that the region under the transformed region is free from any extended defects such as slip that are commonly seen after indentation of $\mathrm{Si}^{21,22}$ Although some defects may exist in the interface region directly under the phase transformation layer, such defects appear highly localized. This is in stark contrast to the large $(\sim 5 \mu \mathrm{m}$ long) extended defects that have been previously observed. ${ }^{24}$ Both selected-area diffraction patterns (SADP) and Raman spectra were used to confirm that the transformed region in Fig. 2(a) is composed of the expected bc8/r8 structure. The SADP shown in Fig. 2(b), taken from the circled region in Fig. 2(a), shows diffraction spots from the bc $8 / \mathrm{r} 8$ phases $^{14}$ as indicated by the arrows. Some a-Si rings are also present, most likely from the thin a-Si layer on the surface caused by ion-beam damage during FIB milling. This can be seen in Fig. 2(a), as $\mathrm{a}-\mathrm{Si}$ is not expected within the transformed zone given the peak indentation strain the sample experiences. ${ }^{20}$ Raman spectra from the residual indent impression (before FIB milling) is shown in Fig. 2(c), from which the peaks associated with the bc $8 / \mathrm{r} 8$ phases $^{7}$ can be clearly seen. A peak from dc$\mathrm{Si}$ at $521 \mathrm{~cm}^{-1}$ is also observed. This is expected to arise from the underlying (untransformed) dc-Si.

The observation of phase transformation without the presence of any other significant form of plastic deformation (such as slip) is a noteworthy result. In addition to phase transformation, all previous studies of spherical indentation in dc-Si have reported other deformation mechanisms ${ }^{29}$ such as the generation of extended defects, including slip bands along the (111) planes $^{14}$ and possible other crystalline defects such as dislocations ${ }^{28}$ (However, it should be noted that dislocations have not been unambiguously identified following room temperature indentation of $\mathrm{dc}-\mathrm{Si}$, although extensive slip and other defects in the crystalline network have previously been clearly visible below the transformed zone. $\left.^{14,18,22,24}\right)$. Dubois et al $^{33}$ propose that crystalline defects not only occur prior to phase transformation but also promote subsequent phase transformation. Thus, the paucity of such defects observed in Fig. 2(a) is clearly of interest. One possible explanation for the phase transformation-only mode observed is that the phase transformed region effectively 'erases' any such defects that were nucleated in the early stages of the indentation loading. Indeed, it has been proposed that such defects are engulfed by the phase transformation precisely because the ( $\beta$-Sn)-Si nucleates from the strain field caused by crystalline defects. ${ }^{34}$ While such a 


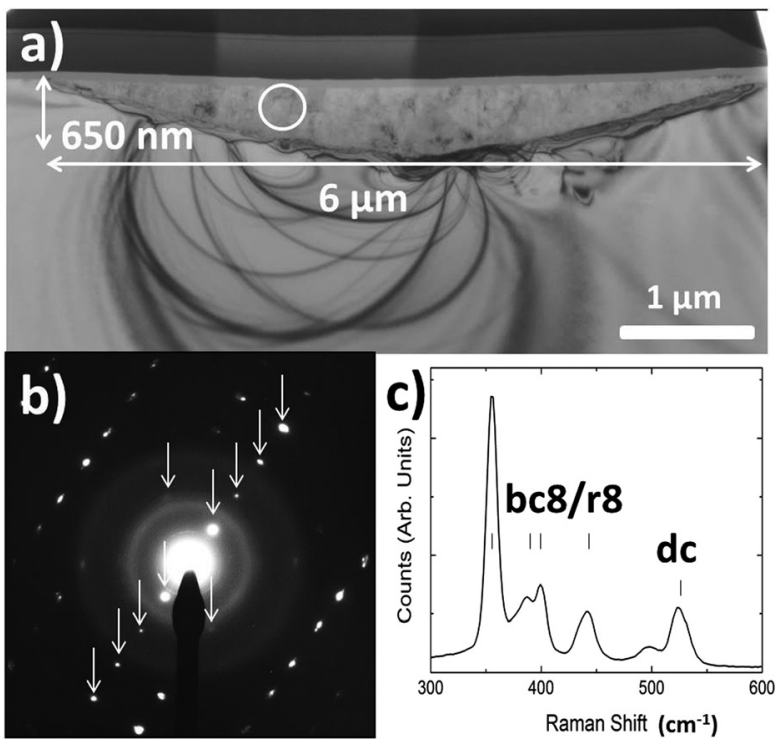

FIG. 2. (a) A BF XTEM image of a residual indent formed with a $500 \mathrm{mN}$ maximum load indentation using a $\sim 21.5 \mu$ m diameter spherical tip. (b) SADP taken from the circled region in (a) with arrows indicating diffraction spots arising from the bc $8 / \mathrm{r} 8$ structure. (c) Raman spectrum of the residual indent impression confirming the presence of the $\mathrm{bc} 8 / \mathrm{r} 8$ phases.

proposal may explain the lack of dislocations as proposed by Saka et al., ${ }^{28}$ slip bands (which are routinely observed and typically penetrate $>5$ times the depth of the transformed zone $^{14}$ ) are clearly too long to be removed by such a process. Thus, it is clear that no slip bands were nucleated during indentation in our case, and phase transformation is essentially the sole form of plastic deformation. This result suggests that phase transformation and crystalline defect nucleation are not intrinsically linked. Instead, it shows that there exists an indentation pressure distribution regime in which a hydrostatic stress-driven phase transformation ${ }^{35}$ can occur while there is still insufficient pressure for the shear stress-driven slip bands/cracking ${ }^{36}$ to also nucleate.

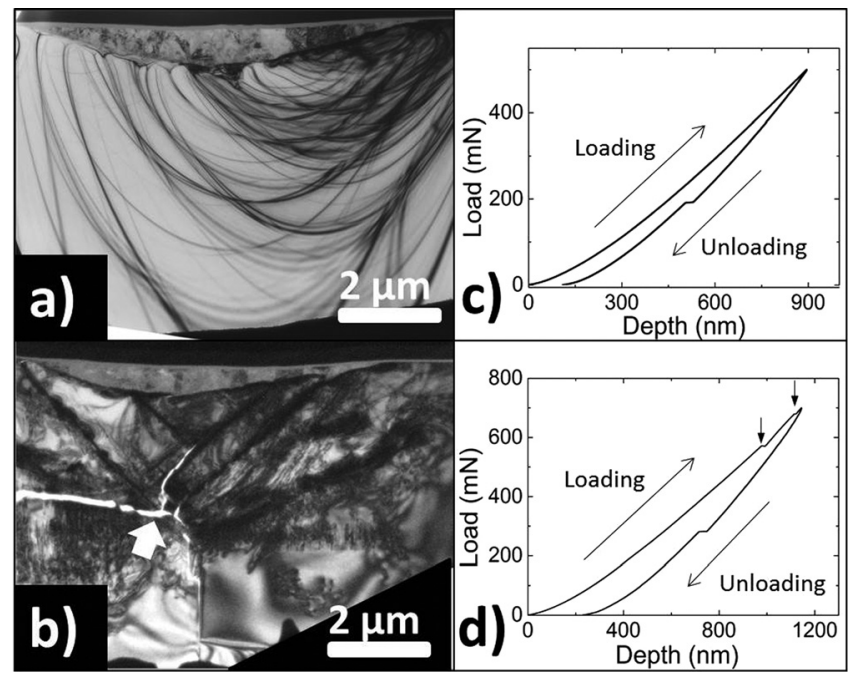

FIG. 3. A BF XTEM of the phase transformed region made via nanoindentation to a maximum load of (a) $500 \mathrm{mN}$ and (b) $750 \mathrm{mN}$. A large subsurface fracture is indicated by the white arrow. The respective load/unload curves can be seen in (c) $500 \mathrm{mN}$ and (d) $750 \mathrm{mN}$ with pop-in events indicated by the arrows. A pop-out event (discontinuity in the unloading section of the curve) is observed in both plots.
Figure 3 contrasts the dramatic difference in the concentration of defects in the underlying substrate below the transformed zone between indents made to maximum loads of $500 \mathrm{mN}$ [Fig. 3(a)] and $750 \mathrm{mN}$ [Fig. 3(b)]. While the crystal matrix under the transformed zone for the $500 \mathrm{mN}$ indent shows very little damage (that is all confined to a transition region, similar to the XTEM image of a separate indent shown in Fig. 2(a)), major slip, cracking, and extensive crystalline damage are evident in the XTEM image of the indent loaded to $750 \mathrm{mN}$. This extensive damage extends to least $5 \mu \mathrm{m}$ beneath the surface. Further, the indentation load curve of the $750 \mathrm{mN}$ indent [Fig. 3(d)] differs from the $500 \mathrm{mN}$ indent curve [Fig. 3(c)] in that it exhibits discontinuities (known as pop-ins) at $\sim 600$ and $\sim 730 \mathrm{mN}$. Pop-in events during spherical indentation are indicative of physical changes in the material that may occur during loading. For example, it has been previously suggested that pop-in events during indentation of $\mathrm{Si}$ tend to occur when the sample first experiences a pressure of $11 \mathrm{GPa}$, which is the threshold for the dc-Si to the $\beta$-Sn phase transition. ${ }^{37}$ However, in this work, Elastica ${ }^{\circledR}$ calculates that $11 \mathrm{GPa}$ is reached in the center of the indent at a load of $\sim 200 \mathrm{mN}$, a load at which no pop-in event is observed. This suggests that a pop-in does not necessarily indicate transformation to the metallic phase but, as suggested previously, ${ }^{22}$ it can indicate the subsequent extrusion of the metallic phase from underneath the indenter tip. Thus, no pop-in is observed during the current study due to the confinement of the transformed region by the indenter tip and the underlying substrate with no material extrusion. Instead, the pop-ins observed in Fig. 3(d) may indicate either cracking $^{37,38}$ or the sudden formation of slip bands. ${ }^{22}$ It is noteworthy that discontinuity events during unloading (known as pop-outs) are also observed in Figs. 3(c) and 3(d) at loads of $\sim 200$ and $\sim 300 \mathrm{mN}$, respectively. These have been previously reported to indicate the sudden transformation of the $\beta$-Sn phase to the $\mathrm{r} 8 / \mathrm{bc} 8$ mixed phase. ${ }^{39}$

It is also interesting to note that the $\sim 6 \mu \mathrm{m}$ wide, $\sim 650 \mathrm{~nm}$ deep volume of the transformed zone shown in Fig. 3(a) is larger than the $\sim 3.5 \mu$ m wide, $\sim 350 \mathrm{~nm}$ deep volume of the higher maximum load indentation shown in Fig. 3(b). While this could be attributed to the differences in the location of the cross-section, much care was taken to ensure that both lamellae were positioned in the centre of the indent. As both lamellae are aligned in the same plane, this difference also cannot be attributed to preferential transformation along a particular crystallographic plane, ${ }^{20}$ and hence, this difference must be addressed. A possible explanation for this surprising result could be that slip/defect propagation and phase transformation are mutually exclusive and competing deformation mechanisms, with one or the other process initiating plastic deformation under particular loading conditions. Indeed, we find that indenting to loads less than $500 \mathrm{mN}$ results in either phase transformation or slip/defect nucleation and propagation, but not both. ${ }^{40}$ This behaviour suggests that the particular deformation process (phase transformation or slip) is stochastic in nature. Thus, it is possible that in the case of the $750 \mathrm{mN}$ indent shown in Fig. 3(b), the nucleation and propagation of crystalline defects is the first deformation process to occur [unlike the indent shown in Fig. 2(a), where the first deformation mechanism is phase 
transformation]. Subsequent increased loading supplies sufficient additional pressure to induce phase transformation as a secondary mode of deformation. Hence, it is plausible that the volume of phase transformation is less than that observed for the indents loaded to $500 \mathrm{mN}$ (where essentially no indentation energy was used to create significant crystalline defects).

In summary, it has been shown that it is possible to engineer a mixed bc8/r8 structure in dc-Si via indentation with minimal damage to the underlying substrate via indentation. Such a transformed zone, formed via indentation to a maximum load of $500 \mathrm{mN}$ using a $21.5 \mu \mathrm{m}$ diameter tip, is $6 \mu \mathrm{m}$ wide and $650 \mathrm{~nm}$ deep and opens up exciting possibilities for exploiting these phases for both optical and electrical applications.

This work was performed in part at the ACT node of the Australian National Fabrication Facility, a company established under the National Collaborative Research Infrastructure Strategy to provide nano and micro-fabrication facilities for Australian researchers. We also acknowledge the ACT node of the Australian Microscopy and Microanalysis Research Facility for use of the TEM facilities. J.E.B. would like to acknowledge the Australian Research Council for a Future Fellowship and funding under the Discovery Project scheme. B.H. gratefully acknowledges funding from an Alvin M. Weinberg Fellowship (ORNL) and the Spallation Neutron Source (ORNL), sponsored by the U.S. Department of Energy, Office of Basic Energy Sciences.

${ }^{1}$ M. Green, "Silicon photovoltaic modules: a brief history of the first 50 years," Prog. Photovoltaics: Res. Appl. 13, 447-455 (2005).

${ }^{2}$ M. Schulz, "The end of the road for silicon?," Nature 399, 729-730 (1999).

${ }^{3}$ J. Crain, G. Ackland, J. Maclean, R. Piltz, P. Hatton, and G. Pawley, "Reversible pressure-induced structural transitions between metastable phases of silicon," Phys. Rev. B 50, 13043 (1994).

${ }^{4}$ J. Hu, L. Merkle, C. Menoni, and I. Spain, "Crystal data for high-pressure phases of silicon," Phys. Rev. B 34, 4679 (1986).

${ }^{5}$ R. Piltz, J. Maclean, S. Clark, G. Ackland, P. Hatton, and J. Crain, "Structure and properties of silicon XII: A complex tetrahedrally bonded phase," Phys. Rev. B 52, 4072 (1995).

${ }^{6} \mathrm{G}$. Pharr, W. Oliver, and D. Harding, "New evidence for a pressureinduced phase transformation during the indentation of silicon," J. Mater. Res. 6, 1129-1130 (1991).

${ }^{7}$ A. Kailer, Y. Gogotsi, and K. Nickel, "Phase transformations of silicon caused by contact loading," J. Appl. Phys. 81, 3057-3063 (1997).

${ }^{8}$ V. Domnich, Y. Gogotsi, and S. Dub, "Effect of phase transformations on the shape of the unloading curve in the nanoindentation of silicon," Appl. Phys. Lett. 76, 2214-2216 (2000).

${ }^{9}$ S. Ruffell, K. Sears, J. Bradby, and J. Williams, "Room temperature writing of electrically conductive and insulating zones in silicon by nanoindentation," Appl. Phys. Lett. 98, 052105 (2011).

${ }^{10} \mathrm{~B}$. Malone, J. Sau, and M. Cohen, "Ab initio study of the optical properties of Si-XII,” Phys. Rev. B 78, 161202 (2008).

${ }^{11} \mathrm{~B}$. Malone, J. Sau, and M. Cohen, "Ab initio survey of the electronic structure of tetrahedrally bonded phases of silicon,” Phys. Rev. B 78, 035210 (2008).

${ }^{12}$ S. Ruffell, K. Sears, A. Knights, J. Bradby, and J. Williams, "Experimental evidence for semiconducting behavior of Si-XII," Phys. Rev. B 83, 075316 (2011).

${ }^{13}$ S. Minomura and H. Drickamer, "Pressure induced phase transitions in silicon, germanium and some iii-v compounds," J. Phys. Chem. Solids 23, 451-456 (1962).

${ }^{14}$ J. Bradby, J. Williams, J. Wong-Leung, M. Swain, and P. Munroe, "Transmission electron microscopy observation of deformation microstructure under spherical indentation in silicon,” Appl. Phys. Lett. 77, 3749-3751 (2000).

${ }^{15}$ B. Haberl, J. Bradby, S. Ruffell, J. Williams, and P. Munroe, "Phase transformations induced by spherical indentation in ion-implanted amorphous silicon," J. Appl. Phys. 100, 013520 (2006).

${ }^{16} \mathrm{~T}$. Juliano, Y. Gogotsi, and V. Domnich, "Effect of indentation unloading conditions on phase transformation induced events in silicon," J. Mater. Res. 18, 1192-1201 (2003).

${ }^{17}$ B. Haberl, M. Guthrie, S. Sinogeikin, G. Shen, J. Williams, and J. Bradby, "Thermal evolution of the metastable r8 and bc8 polymorphs of silicon," High Pressure Res. 35, 99-116 (2015).

${ }^{18}$ S. Ruffell, B. Haberl, S. Koenig, J. Bradby, and J. Williams, “Annealing of nanoindentation-induced high pressure crystalline phases created in crystalline and amorphous silicon,” J. Appl. Phys. 105, 093513 (2009).

${ }^{19}$ S. Ruffell, J. E. Bradby, and J. S. Williams, "Annealing kinetics of nanoindentation-induced polycrystalline high pressure phases in crystalline silicon,” Appl. Phys. Lett. 90, 131901 (2007).

${ }^{20}$ Y. Gerbig, S. Stranick, and R. Cook, "Direct observation of phase transformation anisotropy in indented silicon studied by confocal raman spectroscopy,” Phys. Rev. B 83, 205209 (2011).

${ }^{21}$ I. Zarudi, L. Zhang, and M. Swain, "Microstructure evolution in monocrystalline silicon in cyclic microindentations," J. Mater. Res. 18, 758-761 (2003).

${ }^{22}$ J. Bradby, J. Williams, J. Wong-Leung, M. Swain, and P. Munroe, "Mechanical deformation in silicon by micro-indentation," J. Mater. Res. 16, 1500-1507 (2001).

${ }^{23}$ I. Zarudi, J. Zou, and L. Zhang, "Microstructures of phases in indented silicon: A high resolution characterization," Appl. Phys. Lett. 82, 874-876 (2003).

${ }^{24}$ S. Ruffell, J. Bradby, J. Williams, and P. Munroe, "Formation and growth of nanoindentation-induced high pressure phases in crystalline and amorphous silicon," J. Appl. Phys. 102, 063521 (2007).

${ }^{25}$ Finite Element Software Package Elastica 3.0, Radeberg: ASMEC (2006).

${ }^{26}$ D. M. Longo, J. M. Howe, and W. C. Johnson, "Development of a focused ion beam (fib) technique to minimize $\mathrm{x}$-ray fluorescence during energy dispersive $\mathrm{x}$-ray spectroscopy (eds) of fib specimens in the transmission electron microscope (tem)," Ultramicroscopy 80, 69-84 (1999).

${ }^{27}$ J. E. Bradby, "Nanoindentation-induced deformation of semiconductors," Ph.D. dissertation (The Australian National University, 2002).

${ }^{28}$ H. Saka, A. Shimatani, M. Suganuma, and Suprijadi, "Transmission electron microscopy of amorphization and phase transformation beneath indents in Si," Philos. Mag. A 82, 1971-1981 (2002).

${ }^{29}$ I. Zarudi, L. Zhang, W. Cheong, and T. Yu, "The difference of phase distributions in silicon after indentation with Berkovich and spherical indenters," Acta Mater. 53, 4795-4800 (2005).

${ }^{30}$ J. Field and M. Swain, "Determining the mechanical properties of small volumes of material from submicrometer spherical indentations," J. Mater. Res. 10, 101-112 (1995).

${ }^{31}$ J. Bradby, J. Williams, and M. Swain, "In situ electrical characterization of phase transformations in Si during indentation," Phys. Rev. B 67, 085205 (2003).

${ }^{32}$ D. Williams and C. Carter, The Transmission Electron Microscope (Springer, 1996)

${ }^{33}$ S.-M. Dubois, G.-M. Rignanese, T. Pardoen, and J.-C. Charlier, "Ideal strength of silicon: An ab initio study,” Phys. Rev. B 74, 235203 (2006).

${ }^{34}$ L. Brus, J. Harkless, and F. Stillinger, "Theoretical metastability of semiconductor crystallites in high-pressure phases, with application to $\beta$-tin structure silicon,” J. Am. Chem. Soc. 118, 4834-4838 (1996).

${ }^{35}$ J. Sun, L. Fang, J. Han, Y. Han, H. Chen, and K. Sun, "Phase transformations of mono-crystal silicon induced by two-body and three-body abrasion in nanoscale," Comput. Mater. Sci. 82, 140-150 (2014).

${ }^{36}$ D. Chrobak, K.-H. Kim, K. Kurzydłowski, and R. Nowak, "Nanoindentation experiments with different loading rate distinguish the mechanism of incipient plasticity," Appl. Phys. Lett. 103, 072101 (2013).

${ }^{37}$ T. Juliano, V. Domnich, and Y. Gogotsi, "Examining pressure-induced phase transformations in silicon by spherical indentation and raman spectroscopy: A statistical study,"J. Mater. Res. 19, 3099-3108 (2004).

${ }^{38} \mathrm{R}$. Armstrong, A. Ruff, and H. Shin, "Elastic, plastic and cracking indentation behavior of silicon crystals," Mater. Sci. Eng.: A 209, 91-96 (1996).

${ }^{39} \mathrm{~L}$. Chang and L. Zhang, "Deformation mechanisms at pop-out in monocrystalline silicon under nanoindentation," Acta Mater. 57, 2148-2153 (2009).

${ }^{40} \mathrm{~S}$. Wong, B. Haberl, J. Williams, and J. Bradby, "Exploring the influence of hold time in the onset of plastic deformation in silicon," (to be published). 